\title{
Quasifision timescale: Zeptosecond versus attosecond
}

\author{
A. Ray $^{1}$, A. K. Sikdar ${ }^{1}$ and A. De ${ }^{2}$ \\ ${ }^{1}$ Variable Energy Cyclotron Center, 1/AF Bidhannagar, Kolkata-700064, India \\ ${ }^{2}$ RaniganjGirls' College, Raniganj, Bardhaman- 713358, West Bengal, India
}

\begin{abstract}
Recently a controversy has developed regarding the timescales of quasifission and fission processes of highly excited $\left(E_{X}>50 \mathrm{MeV}\right)$ transuranium and uranium-like nuclei. The mass-angle distributions of quasifission fragments indicate exponential decay law giving timescale of the order of $10^{-21} \mathrm{sec}$ for the quasifission process, whereas for the similar reactions, timescale of the order of $10^{-18} \mathrm{sec}$ were obtained from the crystal blocking technique. In the case of fission of highly excited uranium-like and transuranium nuclei, X-ray-fission fragment coincidence technique gives similar timescales $\sim 10^{-18} \mathrm{sec}$, contradicting much shorter timescales obtained from nuclear techniques. We think the quantum decoherence time of the quasifission/fission decay process could be of the order of $10^{-18} \mathrm{sec}$ and present a quantum mechanical explanation to resolve the puzzle.
\end{abstract}

\section{Introduction}

Although both the fission and quasifission processes have been known for a long time [1], recently a controversy has developed [2] regarding a basic question about the time scales of these nuclear processes. The theories of these processes [1] predict quasifission timescale of the order of $10^{-21} \mathrm{sec}$ and fission timescales of the order of $10^{-20} \mathrm{sec}$ for the highly excited transuranium or uraniumlike complexes, assuming exponential decay in time. Recently R. du Rietz et al. [2] measured mass-angle distributions of ${ }^{64} \mathrm{Ni}+\mathrm{W}$ reaction at $\mathrm{E}\left({ }^{64} \mathrm{Ni}\right)_{\mathrm{Lab}}=310-341$ $\mathrm{MeV}$ and ${ }^{48} \mathrm{Ti}+\mathrm{W}$ reaction at $\mathrm{E}\left({ }^{48} \mathrm{Ti}\right)_{\mathrm{Lab}}=220-260 \mathrm{MeV}$. They performed simulations of the mass-angle distributions parametrizing the nuclear sticking time distribution with a half Gaussian followed by an exponential decay function. The sticking time was converted to the observed scattering angle using calculated angular momentum and moment of inertia of the system. Comparing the simulation with the corresponding experimental mass-angle distribution, they obtained that the timescales for ${ }^{64} \mathrm{Ni}+\mathrm{W}$ and ${ }^{48} \mathrm{Ti}+\mathrm{W}$ quasifission reactions were $5 \times 10^{-21} \mathrm{sec}$ and $10 \times 10^{-21} \mathrm{sec}$ respectively. Andersen et al. [3] measured the timescales of quasifission reactions ${ }^{74} \mathrm{Ge}+\mathrm{W}$ at $\mathrm{E}\left({ }^{74} \mathrm{Ge}\right)_{\mathrm{Lab}}=390 \mathrm{MeV}$, ${ }^{58} \mathrm{Ni}+\mathrm{W}$ at $\mathrm{E}\left({ }^{58} \mathrm{Ni}\right)_{\mathrm{Lab}}=330-375 \mathrm{MeV},{ }^{48} \mathrm{Ti}+\mathrm{W}$ at $\mathrm{E}\left({ }^{48} \mathrm{Ti}\right)_{\text {Lab }}=240-255 \mathrm{MeV}$ by crystal blocking technique and obtained about one attosecond $\left(10^{-18} \mathrm{sec}\right)$ timescale for all those quasifission processes. These results contradict R. du Rietz et al.'s results [2] and theoretical expectations based on nuclear models by more than two orders of magnitude. Fission timescales of highly excited uranium-like nuclei have been found to be of the order of $10^{-20} \mathrm{sec}$ from pre-scission neutron multiplicity measurements [4]. However, all measurements based on crystal blocking [3,5] and atomic X-ray techniques [6-7] have obtained about an attosecond lifetime for the quasifission and fission of highly excited uranium-like and transuranium complexes. Since crystal blocking and atomic X-ray techniques cannot measure lifetimes shorter than $\sim 10^{-19} \mathrm{sec}$, let us examine if these experiments detected only a tiny fraction of the quasifission and fission fragments that might come from the late quasifission/fission processes, as the pre-scission neutron emissions would reduce substantially the excitation energy of the dinuclear complex. Molitoris et al. [6] found from their $\mathrm{X}$-ray-fission fragment coincidence measurement that the fission lifetime of uranium-like nuclei (even at $E_{X}=105 \mathrm{MeV}$ ) was $\geq 4 \times 10^{-18} \mathrm{sec}$ and most of these fission fragments ( $\geq 52 \%$ ) came from such slow fission process. Fregeau et al. [7] produced $\mathrm{Z}=120$ nucleus at a high excitation energy by ${ }^{238} \mathrm{U}+{ }^{64} \mathrm{Ni}$ reaction at $6.6 \mathrm{MeV} / \mathrm{A}$ bombarding energy and found that most of the fission fragments $(>53 \%)$ in the $70 \leq \mathrm{Z} \leq$ 80 region came from slow fission process of lifetime $>$ $2.5 \times 10^{-18} \mathrm{sec}$. Andersen et al. [3] found from crystal blocking experiments that for quasifission reactions such as ${ }^{74} \mathrm{Ge}+\mathrm{W}$ at $\mathrm{E}\left({ }^{74} \mathrm{Ge}\right)_{\mathrm{Lab}}=390 \mathrm{MeV},{ }^{58} \mathrm{~N}+\mathrm{W}$ at $\mathrm{E}\left({ }^{58} \mathrm{Ni}\right)_{\mathrm{Lab}}=350 \mathrm{MeV}$ etc., all the quasifission/ fission fragments came from a slow process of lifetime $\sim 10^{-18}$ sec. So, these experiments indicate that quasifission/ fission processes seem to be primarily very slow processes of lifetime $>10^{-18} \mathrm{sec}$, even for transuranium nuclei produced at a very high excitation energy $\left(\mathrm{E}_{\mathrm{X}}>\right.$ $50 \mathrm{MeV}$ ) and most or all of the quasifission/fission

\footnotetext{
${ }^{1}$ Corresponding author: ray@vecc.gov.in
} 
fragments come from such a slow process. So, clearly there exists a large discrepancy between the absolute values of the quasifission / fission timescales obtained from model-dependent nuclear experiments and significantly less model dependent crystal-blocking/X-ray experiments.

\section{Analysis of experimental results}

We have found that the mass-angle distribution plots of R. du Rietz et al. [2] are consistent with the expected quasifission angular distribution $\left(\frac{d \sigma}{d \theta}\right)_{c . m .} \propto e^{-\frac{\theta_{c . m} .}{\gamma}}$, (where $\theta_{\text {c.m. }}$ is the scattering angle in the center of mass frame) and the analysis presented in ref [2] extracted exponential decay time of the quasifission process. Using 2D color scatter plot given in ref [2], we have made plots as shown in Fig. 1 of $\left(\frac{d \sigma}{d \theta}\right)_{c . m}$ versus $\theta_{\text {c.m }}$ for $\mathrm{M}_{\mathrm{F}}=0.3$ and 04 , where $M_{F}$ is the ratio of the fragment mass to the sum of projectile and target mass for the reaction ${ }^{64} \mathrm{Ni}+\mathrm{W}$ at $\mathrm{E}\left({ }^{64} \mathrm{Ni}\right)_{\mathrm{Lab}}=341 \mathrm{MeV}$. It was found that $\left(\frac{d \sigma}{d \theta}\right)_{c . m}$ versus $\theta_{\text {c.m }}$ curves show Gaussian-like rise and fall pattern for small values of $\theta_{\text {c.m }}$ and $\left(\frac{d \sigma}{d \theta}\right)_{c . m .} \propto e^{-\frac{\theta_{c . m} .}{\gamma}}$ relationship holds good reasonably well for larger values of $\theta_{\text {c.m }}$ implying a quasifission process. Using the linear relationship [8] among the center of mass scattering angle

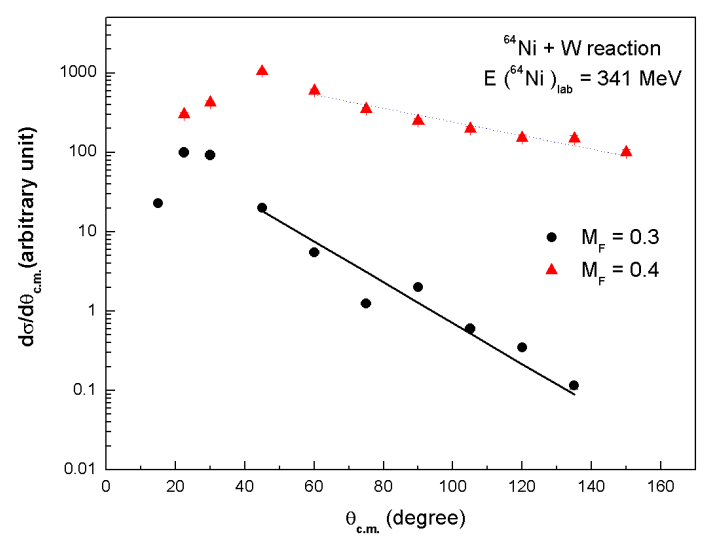

Figure 1. $(\mathrm{d} \sigma / \mathrm{d} \theta)_{\mathrm{c} . \mathrm{m} .}$ vs $\theta_{\text {c.m. }}$ of ${ }^{64} \mathrm{Ni}+\mathrm{W}$ reaction for $\mathrm{M}_{\mathrm{F}}=0.30$ and $\mathrm{M}_{\mathrm{F}}=0.40$.

$\theta_{\text {c.m }}$, angle of rotation $\theta_{\text {rot }}$ and Coulomb deflection angle $\theta_{\text {Coulomb }}$ as well as the relationship $\theta_{\text {rot }}=\left(\frac{L}{I}\right) t$, (valid in the high angular momentum limit) where L, I are orbital angular momentum and moment of inertia of the system and $t$ is the time required to rotate through the angle $\theta_{\text {rot }}$, we obtain from the observed angular distribution an exponential decay law $\sigma(\theta) \propto \frac{d N}{d t} \propto e^{-\lambda t}$, where $\mathrm{N}(\mathrm{t})$ is the number of dinuclear complexes at $\mathrm{t}, \lambda=\frac{1}{\gamma}\left(\frac{L}{I}\right)$ and the lifetime of the exponential decay is $\tau=\frac{1}{\lambda}=\left(\frac{I}{L}\right) \gamma$.
Using experimentally obtained values of $\gamma$ from the observed exponential slopes and the values of the moment of inertia and orbital angular momentum for different values of $\mathrm{M}_{\mathrm{F}}$, we find that the values of dinuclear lifetime increases from $10^{-21} \mathrm{sec}$ to $4.4 \times 10^{-21}$ sec as $\mathrm{M}_{\mathrm{F}}$ increases from 0.25 to 0.45 for ${ }^{64} \mathrm{Ni}+\mathrm{W}$ reaction at $\mathrm{E}\left({ }^{64} \mathrm{Ni}\right)_{\mathrm{Lab}}=341 \mathrm{MeV}$, in good agreement with the results of ref [2]. Similar agreements were also obtained for other reactions. It is expected that the quasifission lifetimes obtained from such analysis and the fission lifetimes of highly excited uranium-like nuclei obtained from pre-scission neutron multiplicity measurements should agree qualitatively with the results obtained from the crystal blocking and X-ray techniques for similar systems. However, crystal blocking experiments gave timescale $\sim 10^{-18} \mathrm{sec}$ for similar reactions and X-ray measurements also gave timescale $\sim 10^{-18} \mathrm{sec}$ for the fission of highly excited uranium-like and transuranium nuclei. Assuming a very high value of nuclear viscosity parameter, Andersen et al. [3] considered the dynamics of quasifission as a diffusion process and obtained $10^{-18} \mathrm{sec}$ lifetime for the process. However, a large value of nuclear viscosity parameter should imply a large exponential damping constant $(\gamma)$ and it should lead to a symmetric angular distribution of the quasifission products i.e. $\left(\frac{d \sigma}{d \theta}\right)_{c . m}=$ const, contrary to the experimental results (as shown in Fig. 1). In the case of highly excited uranium-like and transuranium nuclei, most of the fission fragments come from the decay of the highly excited compound nucleus, even after considering neutron emission from the compound nucleus and the expected fission timescale should be of the order of $10^{-20}$ sec. So, the observed large discrepancies between the measured lifetimes of the quasifission/fission processes obtained by using non-nuclear and nuclear techniques are unlikely to be due to any nuclear effect such as fission dynamics or neutron emissions.

\section{Quantum mechanical analysis}

We have found from the above analysis that the observed angular distribution of a quasifission process for a specific value of $\mathrm{M}_{\mathrm{F}}$ indicates emission of the fragments with an exponential decay law in time. Such an exponential decay law in time implies the formation of quasibound states comprising the fragment and the residual nuclei. It is usually thought that the exponential decay starts from the instant of formation of the dinuclear system. However, the exponential decay law should not be valid from the instant when the initial dinuclear state was formed, because the unitary reversible quantum mechanical time evolution of the unstable states does not lead to irreversible exponential decay. Quantum mechanics predicts an approximately flat initial survival probability of the ensemble of dinuclear systems as long as the quantum coherence i.e. the linear superposition of the dinuclear state and fissioning state is maintained. Hence, there should be an approximately flat initial survival probability of the ensemble of dinuclear systems for small values of $\mathrm{t}$. So, any model-independent direct 
measurement (crystal blocking or X-ray technique) of the lifetime of the quasifissioning/fissioning complex should measure the sum of the quantum mechanical delay time and the lifetime of the exponential decay and hence the measured lifetime would be longer than the exponential lifetime measured by nuclear techniques [2]. The time evolution of the initial dinuclear state produces a superposition of dinuclear and fissioned states and very little irreversible fission decay takes place as long as the system remains in the coherent superposition of states. So, the angular distributions of fragments (obtained from the MAD plots) do not have any information about the time that the system had spent in the coherent superposition of states, because no measurable fission decay took place during that time.

Let us consider the entire ion as a quantum detector observing the nuclear fission/quasifission process. The nuclear system at first couples with the ion having vacancies in the atomic orbitals and after the loss of quantum coherence of the coupled system, classical descriptions and exponential decay law are applicable. Let $\mathrm{H}$ be the Hamiltonian of the nuclear system. Then, the time evolution of the unstable decaying dinuclear state $\left(\left|\psi_{\text {dinuclear }}\right\rangle\right)$ comprising a fragment and the residual nuclei should produce the following superposition of states.

$$
\begin{gathered}
e^{-\frac{i H t}{\hbar}}\left|\psi_{\text {dinuclear }}>=\sum_{i} \alpha_{i}(t)\right| \psi_{\text {fission }}>{ }_{i} \\
+\beta(t) \mid \psi_{\text {dinuclear }}>
\end{gathered}
$$

where $\mid \psi_{\text {fission }}>_{i}$ indicate different macroscopically distinguishable fission fragment states. These states should be orthogonal to one another for different values of $\mathrm{i}$ and $\mathrm{j}$ and also with the dinuclear state. It can be shown $[9,10]$ that

$$
\begin{gathered}
\beta\left(t+t^{\prime}\right)=\beta(t) \beta\left(t^{\prime}\right) \\
+\sum_{i} \alpha_{i}(t)\left\langle\psi_{\text {dinuclear }}\left|\exp \left(-i H t^{\prime}\right)\right| \psi_{\text {fission }}\right\rangle_{i}
\end{gathered}
$$

and $\beta(t) \propto \exp (-\lambda t)$ with $\operatorname{Re}(\lambda)>0$, only when the reformation amplitude of the unstable state $\psi_{\text {dinuclear }}$ becomes zero. However, it can never be zero from the solution of the time dependent Schrodinger equation. Hence, the condition for the start of the exponential decay and the classical description of the process is the loss of the quantum coherence of the nuclear state as a result of the interaction of the nuclear system with the ion and environment.

In nuclear quasifission or fission process, a target atom is bombarded by a projectile ion producing a combined ion whose nucleus becomes a compound or dinucleus. The incoming projectile has different probabilities to produce vacancies in different atomic orbitals of the target atom. So, the excited combined ion might be represented by the following coherent superposition of all the atomic orbitals:

$\mid A^{*}>=\sum_{i} C_{i} A_{i}^{*}$, where $A_{i}^{*}$ indicates atomic wave function with one vacancy in $i^{\text {th }}$ orbital. Since even for zero impact parameter collision, it is much more likely to produce a vacancy in the outer electronic orbital than inner orbital [11], the magnitude of $C_{i}$ increases rapidly for outer electronic orbitals. Let us consider for simplicity the nuclear state as the superposition of the most probable fission fragment state and the dinuclear state. So

$$
\begin{aligned}
e^{-\frac{i H t}{\hbar}} \mid \psi_{\text {dinuclear }} & >\approx \alpha_{p}(t) \mid \psi_{\text {fission }}>{ }_{p} \\
& +\beta(t) \mid \psi_{\text {dinuclear }}>
\end{aligned}
$$

where $\left|\psi_{\text {fission }}\right\rangle_{p}$ indicates most probable fission fragment pair and $\left\langle\left(\psi_{\text {fission }}\right)_{p} \mid \psi_{\text {dinuclear }}\right\rangle=0$. Let us now consider the coupling of the nuclear state with the ionic state $\left|A^{*}\right\rangle$. The interactions of the dinuclear state and fissioned state with the atomic orbital state $\left|A_{i}^{*}\right\rangle$ containing a vacancy in $i^{\text {th }}$ orbital might be represented as follows:

$\left|\psi_{\text {dinuclear }}>\right| A_{i}^{*}>\rightarrow \gamma_{i}(t)\left|\psi_{\text {dinuclear }}>\right| A_{i}, X_{i}>$ (1) where $\gamma_{i}(t)=1-e^{-\lambda_{i} t}$ and $\lambda_{\mathrm{i}}$ is the decay rate of $i^{\text {th }}$ vacancy as it is filled up from the higher orbitals. Here, $\mid A_{i}, X_{i}>$ denotes an ion with no vacancy in $\mathrm{i}^{\text {th }}$ orbital, but a vacancy in $(i+1)^{\text {th }}$ orbital due to the electronic transition and $\mathrm{X}_{\mathrm{i}}$ denotes the corresponding X-ray photon. Regarding the interaction of $\left|\psi_{\text {fission }}\right\rangle_{p}$ with $\left|A_{i}^{*}\right\rangle$, at very early time, the distant atomic orbital will not experience any significant effect as the fission fragments start separating out and only after a certain time $t_{i}$, the $i^{\text {th }}$ orbital will be destroyed. So for $t<t_{i}$,

$\left|\psi_{\text {fission }}>_{p}\right| A_{i}^{*}>\rightarrow \gamma_{i}(t)\left|\psi_{\text {fission }}>_{p}\right| A_{i}, X_{i}>$ and for $t>t_{i}$,

$\left|\psi_{\text {fission }}>_{p}\right| A_{i}^{*}>\rightarrow\left|\psi_{\text {fission }}>_{p}\right| \bar{A}_{i}, \bar{X}_{i}>$

where $\mid \overline{A_{i}}, \bar{X}_{i}>$ indicates an ion with up to $\mathrm{i}^{\text {th }}$ orbital destroyed as the fission fragments separate out and no corresponding X-ray photon. We have the orthogonality conditions $\left\langle A_{i}, X_{i} \mid A_{j}, X_{j}\right\rangle=0$ for $\mathrm{i} \neq \mathrm{j}$ and $\left\langle A_{i}, X_{i} \mid \bar{A}_{i}, \bar{X}_{i}\right\rangle=$ 0 . So the combined dinuclear-ion state evolves $[12,13]$ into a correlated state

$$
\begin{aligned}
& \left|\psi_{\text {correlated }}>=\alpha_{p}(t) \sum_{i} C_{i}\right| \psi_{\text {fission }}>{ }_{p} \mid \bar{A}_{i}, \bar{X}_{i}> \\
& +\beta \sum_{i} C_{i} \gamma_{i}(t)\left|\psi_{\text {dinuclear }}>\right| A_{i}, X_{i}>
\end{aligned}
$$

where the summation is over all the atomic orbitals. The minimum time required to evolve into this correlated state is $t_{n}$, where $t_{n}$ is the time required for the destruction of the outermost $\mathrm{n}^{\text {th }}$ orbital of the ion due to the fission of the nucleus. The corresponding density matrix is $\rho^{c}=$ $\left|\psi_{\text {correlated }}><\psi_{\text {correlated }}\right|$ and after tracing over the environment [13], one gets the reduced density matrix $\rho^{r}=$

$$
\begin{aligned}
& \left.\left|\alpha_{p}\right|^{2} \sum_{i=1}^{n}\left|C_{i}\right|^{2}\left|\psi_{\text {fission }}>_{p}<\psi_{\text {fission }}\right|\right|_{p}\left|\overline{A_{i}}, \bar{X}_{i}><\bar{A}_{i}, \bar{X}_{i}\right| \\
& +|\beta|^{2} \sum_{i=1}^{n}\left|C_{i}\right|^{2}\left|\gamma_{i}\right|^{2}\left|\psi_{\text {dinuclear }}><\psi_{\text {dinuclear }}\right| \mid A_{i}, X_{i}> \\
& <A_{i}, X_{i} \mid
\end{aligned}
$$

The reduced density matrix contains only classical correlations implying that the system would be either in the fissioned state or dinuclear state and the exponential decay of the dinuclear state would start. Since an ion is a much bigger object than a nucleus, decoherence time of the nuclear-ion system due to its interaction with the environment (i.e. tracing over the environment) should be much faster than decoherence time of the fission/quasifission process. So, we might consider the time required to transform from $\rho^{c}$ to $\rho^{r}$ as instantaneous 
and take the time $t_{n}$ required to form the coupled state with all the orbitals of the ion (as given in eq. (4)) as the decoherence time of the fissioning nucleus. It is very important to couple with all the atomic orbitals, because otherwise the reduced density matrix will contain quantum correlations and decoherence will not be achieved. We find from eq. (1) and eq. (2) that for $\mathrm{t}<t_{i}$, the interactions of both $\left|\psi_{\text {dinuclear }}\right\rangle$ and $\left|\psi_{\text {fission }}\right\rangle_{p}$ with $\left|A_{i}^{*}\right\rangle$ produce the same state $\left|A_{i}, X_{i}\right\rangle$, whereas for $t>t_{i}$, the interactions of fissioned and dinuclear states on $\left|A_{i}^{*}\right\rangle$ produce orthogonal states. If the ion contains ' $\mathrm{n}$ ' number of orbitals, then for $t>t_{n}$, the interactions of the dinuclear and fissioned states with all the orbitals will produce mutually orthogonal states and at that point, the interaction of coupled system with the environment will produce a reduced density matrix containing only classical correlations. So, the question is what is the estimate for $t_{n}$ ? It is known [14] that the electronic binding energy would decrease as the separation between the fission fragments would increase and eventually the original orbitals would be completely destroyed. For an order of magnitude estimate of $t_{n}$, taking the speed of fission fragments as $\sim 10^{9} \mathrm{~cm} / \mathrm{sec}$ and the radius of a transuranium atom as $\sim 10^{-8} \mathrm{~cm}, t_{n}<$ $10^{-17} \mathrm{sec}$, because by that time the fission fragments have crossed the radius of the transuranium atom, all the atomic orbitals are expected to be destroyed. Hence we might consider $t_{n}$ is of the order of $10^{-18} \mathrm{sec}$. The classical exponential decay of the nuclear system should start after the loss of quantum coherence in attosecond time scale and very little fission decay would occur before that. So, any direct measurement (such as crystal blocking or Xray technique) of the quasifission/fission timescale should measure the sum of quantum decoherence time and the classical exponential decay time $\approx 10^{-18} \mathrm{sec}+10^{-21} \mathrm{sec}$ $\approx 10^{-18}$ sec. Qualitatively speaking, the interaction of the dinuclear state with the atomic states producing photons is like a measurement process on the dinuclear state starting from $\mathrm{t}=0$ instant when the dinuclear state was formed. This measurement process is quantum Zeno effect inhibiting [10] the time evolution of the dinuclear state. We consider the timescale of creation of fully correlated nuclear-atom wave function as decoherence time of the dinuclear state and this is the timescale of destruction of all the atomic orbitals when no further interaction between nuclear and atomic states is possible.

\section{TDHF calculation}

Time dependent Hartree-Fock calculations (TDHF) [1516] apparently show that the quasifissioning fragments separate out in zeptosecond $\left(\sim 10^{-21} \mathrm{sec}\right)$ timescale. However in TDHF calculations of heavy ion collisions, the relative motion between the nuclei is treated classically while the internal degrees of freedom of nucleons and their couplings to collective excitations are treated quantum mechanically on a mean field level described by a single Slater determinant. TDHF calculations consider time evolution of an initial state wave function that is a product of ground state wave functions of two nuclei boosted with relative velocity. After collision, single particle wave functions extend spatially and the final stage wave function remains a superposition of states with different particle number distributions. Quantum mechanically, nothing conceivable as decay can take place as long as the superposition of states persists. However, TDHF calculations use classical trajectories and impact parameters and for collisions outside the fusion critical impact parameter, the fragments are assumed to separate out irreversibly, although the final state wave function remains a superposition of states. In other words, quantum decoherence time is not included in TDHF calculations and the separation time of the fragments is essentially based on classical considerations.

\section{Conclusion}

In summary, the controversy regarding the quasifission/fission timescale is probably because the nuclear experiments such as mass-angle distributions measure the exponential decay time of the dinuclear system, whereas crystal blocking/X-ray experiments measure the total lifetime that is a sum of quantum decoherence time and exponential decay time. TDHF codes give classical contact time of the two nuclei on the basis of trajectory calculations and quantum decoherence time is not included in any such calculations. Our quantum mechanical analysis shows that the quantum decoherence time could be of the order of $10^{-18} \mathrm{sec}$ for the quasifission/fission process and the consideration of quantum decoherence time might resolve the controversy regarding the lifetime of quasifission process.

\section{References}

1. W. U. Schroder, J. R. Huizenga, Treatise on HeavyIon Science (D. A. Bromley,1984)

2. R. du Rietz et al., Phys. Rev. Lett. 106, 05271 (2011)

3. J. U. Andersen et al., Phys. Rev. C 78, 064609 (2008)

4. D. J. Hinde et al., Phys. Rev. C 45, 1229 (1992)

5. F. Goldenbaum et al., Phys. Rev. Lett. 82, 5012 (1999)

6. J. D. Molitoris et al., Phys. Rev. Lett. 70, 537 (1993)

7. M. O. Fregeau et al., Phys. Rev. Lett. 108, 122701 (2012)

8. J. Toke et al., Nucl. Phys. A 440, 327 (1985)

9. L. Fonda et al., Rep. Prog. Phys. 41, 587 (1978)

10. D. Home, Conceptual Foundations of Quantum Physics (plenum press, N.Y., 1997)

11. G. Soff et al., Phys. Rev. Lett. 41, 1167 (1978)

12. W. H. Zurek, Los Alamos Science 27, 2 (2002)

13. W. H. Zurek, Rev. Mod. Phys. 75, 715 (2003)

14. J. Reinhardt and W. Greiner, Treatise on Heavy-Ion Science (D. A. Bromley,1984)

15. K. Sekizawa and K. Yabana, Phys. Rev. C 88, 014614 (2013)

16. A. Wakhle et al., Phys. Rev. Lett. 113, 182502 (2014) 